\title{
Factores psicosociales que favorecen la Movilidad Social
}

\author{
Silvia Jiménez Noguera, Marcela de Aguilar, Rafael Román Quirós \\ Universidad de Iberoamérica
}

\section{Resumen:}

La presente investigación se ha propuesto el identificar y analizar distintos factores psicosociales que le han permitido a personas que han nacido y crecido en un barrio urbanomarginal, el lograr en opinión de estos superar su estrato social de origen. Dicha investigación se llevó a cabo con 8 participantes durante el tercer período del año 2014 en la provincia de San José, Costa Rica, bajo un enfoque cualitativo y fenomenológico de la mano de entrevistas semi-estructuradas que se centraban en sus historias de vida. Se identificó en este sentido un espacio familiar de interacción positiva en el cual no se desvaloriza la educación, así como una figura materna que inculca valores, que exige un rendimiento académico y que establece límites. Asímismo se encuentra una influencia negativa de los pares, y una serie de labores y estresores adicionales compartidos en las historias de vida de los participantes, ante los cuales lograron salir resilientes a través de diferentes valores como la perseverancia, constancia, responsabilidad, honestidad y autonomía. Como otros aspectos presentes, se identificó una afinidad por el estudio y la claridad en cuanto a la meta de lograr salir del barrio de origen.

Palabras claves: Movilidad Social, Pobreza, Resiliencia, Red de Apoyo, Educación

\section{Introducción}

La pobreza en nuestro país se ha logrado mantener por más de diez años en una relativa estabilidad que ronda el 20\%; esto gracias a una serie de políticas públicas universales y selectivas (Consejo Centroamericano de Procuradores de Derechos Humanos, 2008), que han colocado a Costa Rica entre las naciones con menor incidencia de pobreza en Latinoamérica. Sin embargo es importante, en primer lugar; tomar en cuenta que si bien se trata de un porcentaje que ha sido estable a través de los años, la población de la región aumenta continuamente, y con ello la cantidad de personas en dicha condición.

En segundo lugar la pandemia mundial del 2020, ha tenido un fuerte impacto sobre la región, lo que implicará un importante empobrecimiento económico, así como un muy probable debilitamiento del aparato institucional costarricense que permita paliar las brechas socioeconómicas y ayudar a los más necesitados.

En esta época de incertidumbre es difícil planificar y pronosticar, sin embargo el Instituto de Investigaciones en Ciencias Económicas (IICE, 2020) de la Universidad de Costa Rica estimó en su estudio titulado "Estimación de los Efectos del COVID-19 sobre la economía costarricense" que la pobreza podría llegar a afectar al 29\% de los hogares del país; esto sin mencionar las cifras del Instituto Nacional de Estadística y Censos (INEC)

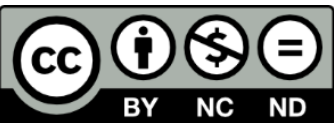

Esta obra está bajo una licencia de Creative Commons Reconocimiento-NoComercial-SinObraDerivada 4.0 Internacional 
publicadas en Julio, en las cuales el desempleo ha alcanzado un 20\% en los meses de marzo, abril y mayo (2020).

La pobreza como fenómeno social comienza a ser estudiada como una operación estadística que afecta el ingreso per cápita. Es durante la década de los años sesenta y setenta que adquirirá un enfoque multidimensional, lo que significa una nueva conceptualización no estrictamente monetaria, que en lugar de tomar en cuenta el poder adquisitivo, centra la atención en estándares de vida observables (Bazán-Ojeda, Quintero-Soto y HernándezEspitia, 2011).

Spicker (1999) identifica once criterios diferentes para determinar la condición de pobreza, incluyendo el ámbito psicológico y social; a saber, 1) por la necesidad de bienes o servicios materiales, 2) un patrón de privación de ciertas necesidades respecto a otras, como por ejemplo techo o comida por encima del transporte, 3) recursos limitados 4) estándar de vida, 5) desigualdad, 6) posición económica, 7) clase social, 8) dependencia (de beneficios sociales por ejemplo), 9) falta de seguridad básica (vulnerabilidad a riesgos sociales), 10) falta de derechos subjetivos (como el no tener acceso a una vivienda o a no poder adquirir alimentos que de hecho existen), 11) exclusión social (no sólo en lo económico, sino en cuanto a la salud, educación, vivienda, y acceso a servicios y a deudas). A su vez, Spicker agrupa los once criterios en 3 grandes categorías; a) por condiciones materiales (1, 2 y 3 ), b) por circunstancias económicas $(4,5,6)$ y c) por posición social.

Respecto a los estratos sociales, éstos cuentan con varias definiciones dependiendo de la línea sociológica que se estudie. Sin embargo en el presente estudio se conceptualiza como:

la división de los miembros de una sociedad en una jerarquía de distinta clase de status o estrato; de manera que los miembros de cada clase tengan relativamente el mismo status y los miembros de las otras clases tengan menor o mayor status. El status o estrato es la evaluación relativa de los miembros de cada clase social vinculado a dos factores clave: el poder y el prestigio (Universidad Nacional de Nordeste, 2010, p.1)

Respecto a las teorías sociológicas existentes en la actualidad para explicar el fenómeno de la pobreza, Brady (2019) agrupa a las mismas en tres familias teóricas; las teorías conductuales (centradas en la conducta individual que es motivada por la cultura o por los incentivos), las estructurales (centradas en aspectos demográficos dentro del mercado laboral que dan como resultado tanto conductas como situaciones de pobreza) y las políticas (centradas en el poder y las instituciones). Sin embargo, vale aclarar que la pobreza como un fenómeno de estudio en las Ciencias Sociales no cuenta con una teoría clara en cuanto a sus causas (Brady, 2019), sino que las investigaciones en el tema suelen tener en mayor medida un abordaje descriptivo (Desmond y Western, 2018).

Esta creciente cifra en nuestra situación actual aunada al desempleo en el país, dificultan aún más el que personas provenientes de lugares urbano-marginales logren sobreponerse a sus necesidades a través de un proceso de movilidad social. Munist, Santos, Klotibrenco, Suárez, Infante y Grotberg (1998) definen un barrio marginal como aquel que

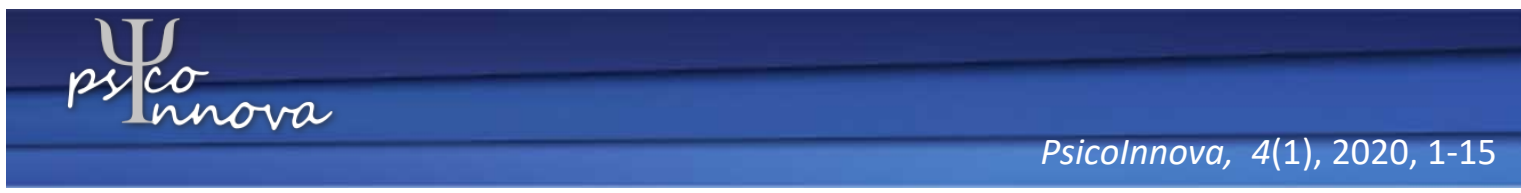


carece de una o más de las siguientes condiciones: acceso a agua potable, a sanidad, a espacios habitables suficientes, a una vivienda construida con material sólido, riesgo mínimo de conductas como delincuencia, drogadicción y prostitución.

Entendemos por movilidad social, la capacidad de un grupo de la sociedad o de una persona, para cambiar su posición dentro del sistema social, ante lo cual toman parte una serie de condiciones personales; sobretodo el nivel de ingresos, el nivel educativo, o la profesión (Fernández-Guzmán, 2019). Más propiamente, la movilidad intergeneracional se determina comparando la posición de los progenitores del individuo, respecto a la posición de él mismo en una misma edad determinada, de manera que obtendríamos una movilidad social ya sea ascendente, en la cual se alcanza una promoción social, o descendente, en la cual el resultado es una degradación social (Sokołowska, 2014).

En todo caso, este proceso tiene consecuencias en la felicidad de las personas (Aldridge, 2003), en el acceso material de las mismas y en cuanto a su estado de salud (Adams, White, Pearce y Parker, 2004; Boyle, Norman y Popham, 2009; Smith, Hart, Watt, Hole y Hawthorne, 1998), todo lo cual fundamenta la importancia de una investigación como esta, en la cual se intenta dilucidar aquellas características personales que contribuyen a este proceso.

En este sentido, sabemos que los factores sociales, materiales, culturales, físicos y sobretodo educacionales (Neelsen, 1975) en la niñez o la juventud temprana, tienen una fuerte influencia en cuanto a la dirección (ascendente o descendente) que pueda tener la movilidad social en la adultez (Blane, Smith y Hart, 1999).

En cuanto al aspecto educativo propiamente, existe un vínculo importante y creciente entre los logros educacionales y logros ocupacionales (Peña-Leiva, 2010), así como entre el nivel educativo y la movilidad social, razón por la cual la educación pública resulta esencial para romper las inercias entre orígenes y destinos (Huerta, 2012).

El Sistema de Información de Tendencias Educativas en América Latina (SITEAL) en un informe sobre la Transmisión Intergeneracional de las Desigualdades Educativas basado en las encuestas nacionales en hogares en la década de los años noventa en Argentina, Brasil, Chile, Costa Rica, Honduras y México, logra determinar que el mínimo de años de estudio para haber podido acceder a condiciones de vida digna es de 10 años, lo cual a su vez tiene un fuerte impacto en la calidad de vida (SITEAL, s.f.). Este aspecto educativo de la movilidad social en nuestro país fue estudiado preliminarmente por Pérez (2004), quien encuentra una relación en cuanto al nivel educativo alcanzado por los padres, y el cómo este influencia a sus hijos, en el tanto la mayoría de los hijos (un 66\%) de padres sin educación formal alcanzan la primaria; el 39\% de los hijos de padres con educación primaria alcanzan la secundaria, y un $32 \%$ de los hijos de padres con educación secundaria, alcanzan el grado universitario.

Otro aspecto relacionado a la movilidad social dentro de la literatura, es el del apoyo social que una persona percibe dentro de su familia o grupo de amigos (Ahluwalia, Dodds y Baligh, 1998). Estas redes de apoyo surgen como estrategias para cubrir necesidades diversas, y consisten en un flujo de intercambios entre familia, amigos, y miembros de una comunidad (Sagrario, Montes de Oca y Arroyo, 2019), beneficiando al individuo desde una 
perspectiva moral, económica o social (Lomnitz, 1981) que contribuye al sentido de pertenencia del mismo, reduciendo el estrés, dotando en general al individuo de mejores condiciones de afrontamiento (Ahluwalia et al, 1998) y permitiéndole mayores probabilidades para hacer realidad el proyecto de vida propio. Estos apoyos pueden ser materiales (monetarios o no monetarios como en el caso de la comida, pagos de servicios, etc), instrumentales (transporte, quehaceres del hogar), emocionales (con afecto) o cognitivos (con la transmisión de experiencias o conocimientos) (Guzmán, Huenchuan y Montes de Oca, 2003).

Otro aspecto esencial dentro del tema es el de la resiliencia; un concepto proveniente de la física, referido a la capacidad de un material determinado para volver a su forma original después de haber sufrido altas presiones (Parker, 1991). El término, que proviene del latín "resilio"; volver atrás, volver de un salto, o rebotar, fue posteriormente adaptado a las Ciencias Sociales (Munist et al, 1998) para caracterizar el proceso mediante el cual las personas logran desarrollarse en armonía y normalidad con su medio a pesar de que estas se encuentren en un contexto socioculturalmente deprivado y desfavorecido, y a pesar de que hayan experimentado desde su niñez, situaciones conflictivas (Luthar y Cicchetti, 2000; Werner, 1984).

Bonanno, Westphal y Mancini (2011), definen la resiliencia como la habilidad que tienen los adultos que son expuestos a eventos aislados y potencialmente disruptivos, como lo puede ser un hecho violento o amenazante para la propia vida, o la muerte de una relación cercana, para lograr una estabilidad relativa y niveles sanos de funcionamiento físico y psicológico, tal como sucede con los participantes de la presente investigación, cuyos orígenes se encuentran en barrios urbano-marginales en los cuales convivían con situaciones de alto riesgo; logrando sin embargo, desarrollarse de manera sana y exitosa (Rutter, 1993)

González, Valdez y Zavala (2008) ahondaron en distintos factores resilientes en adolescentes mexicanos de entre 14 y 18 años de edad, encontrando ciertos elementos presentes, como seguridad personal, baja autoestima, afiliación, altruismo y familia. Se encontró asimismo una diferencia significativa en los análisis por sexo, en donde los hombres se mostraron más resilientes con rasgos de ser más independientes, mientras que las mujeres lograban ser resilientes siempre y cuando exista un apoyo externo significativo.

Peña-Leiva (2010) en un estudio sobre competencias y factores psicosociales de universitarios resilientes de bajo origen socioeconómico; encuentra a nivel personal una serie de características como un auto-concepto positivo, visión de futuro y autorreflexión, y el establecimiento de estrechas relaciones interpersonales. En un nivel psicosocial, los participantes señalaban el haber tenido profesores afectuosos y exigentes en la niñez, una buena calidad académica, un ambiente familiar afectuoso, padres exigentes que establecían límites, con una visión de los estudios como algo que lleva a una mejor calidad de vida, y quienes a pesar de no tener estudios formales, eran percibidos como cultos y como personas que inculcaban valores.

En Costa Rica, Villalobos-Cano (2009) ha desarrollado el Inventario de Cualidades Resilientes para Adolescentes (ICREA) con el que se encontraron diferencias significativas entre géneros, en el tanto los hombres resultaron ser más auto-eficaces respecto a las mujeres,

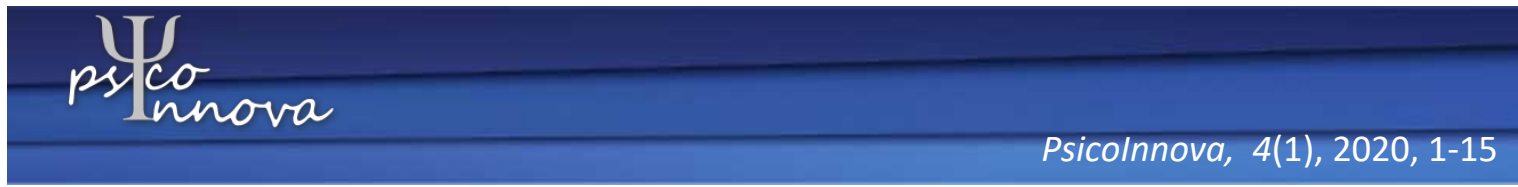


quienes utilizaban en mayor medida estrategias evasivas. Asímismo, López (2010) analiza la relación entre factores de resiliencia y rendimiento académico en alumnos adolescentes de instituciones educativas ubicadas en contextos de alta vulnerabilidad social. Los resultados indican que la relación entre resiliencia y los logros de aprendizaje se fortalece en dos situaciones de adversidad que reportan los adolescentes: divorcio o separación de sus padres, y embarazo propio o de la pareja.

Por último, existen por lo demás factores exógenos que deben ser considerados dentro de la movilidad social; tales como los cambios en el mercado laboral, las oportunidades laborales que el contexto ofrece, la distribución de la riqueza (Ong Puay Tee, 2007) o un contexto económico que es producto de una pandemia. En ese sentido se espera que este trabajo sea de valor y motivación para todos aquellos interesados en trascender un contexto difícil.

\section{Método}

\section{Participantes.}

La presente investigación se ha planteado entrevistar a 8 adultos de nacionalidad costarricenses ( 3 mujeres y 5 hombres) quienes han nacido y vivido en un barrio urbano marginal, que han cursado una carrera profesional y que consideran haber logrado moverse de manera ascendente en cuanto al estrato social, y que cuentan con más de un año de estar laborando. Adicionalmente, de entre los participantes, el entrevistado con menores ingresos mensuales recibía poco más de 350 mil colones mensuales. Todos los participantes leyeron y firmaron un consentimiento informado, en el cual fueron debidamente informados de la investigación y del carácter voluntario de la misma garantizándoles a su vez el anonimato de los datos proveídos.

\section{Instrumentos.}

El trabajo con los participantes fue llevado a cabo con entrevistas semiestructuradas consistentes en una guía de preguntas en las que las entrevistadoras contaban con la libertad de adicionar interrogantes que les permitiera obtener más información o aclarar la misma, en caso de considerarlo necesario (Hernández, Fernández y Baptista, 2014).

\section{Procedimientos de Recolección.}

Las entrevistas fueron llevadas a cabo con cada participante de manera individual y de manera posterior a la lectura y firma del consentimiento informado. Finalmente se procedió a la transcripción de las grabaciones. 


\section{Procedimientos de análisis.}

La presente investigación se ha realizado bajo un enfoque cualitativo (Mason, 2018), y un alcance de tipo exploratorio (Hernández, Fernández, Baptista, 2014); esto se refiere de manera concreta, a identificar características, y factores comunes en diferentes personas que consideran haber logrado movilidad social ascendente, para lo cual se ha partido de un diseño fenomenológico de investigación en el que se empleó un Análisis de Contenido como método de análisis.

\section{Resultados}

A partir de las entrevistas efectuadas se presentan a continuación los resultados para 5 categorías psicosociales, a saber; educación, familia, pares y amigos, trabajo y características personales. Las mismas se presentan a continuación:

\section{Educación.}

En primer lugar, encontramos que es común a todos los participantes el haber estudiado en escuelas públicas debido a la escasez de recursos. A pesar de tratarse de educación pública, los entrevistados relatan que los materiales de estudio como libros, cuadernos y uniformes fueron de difícil acceso. También mencionan que la calidad de la educación y el apoyo recibido por parte de los docentes fue de calidad y de vital importancia para su progreso escolar. Como menciona una de las entrevistadas:

"Yo tenía una maestra que se preocupaba por mi superación, porque entendía mi entorno social y lo mucho que se le dificultaba a personas como yo, salir adelante".

Para la mayoría de los entrevistados, la familia o impulsó la culminación de los estudios, o bien, al menos no se involucró negativamente. Los entrevistados mencionan que mantenerse en el sistema educativo durante la época de colegio fue particularmente difícil, debido al nivel de dificultad de los contenidos de estudio y a la influencia negativa de los pares.

Con respecto a los estudios universitarios, los entrevistados comentan que este proceso resultó "complicado" ya que para ese momento debían cargar con varias responsabilidades más allá de los estudios. Entre dichas responsabilidades se mencionó el tener que trabajar, atender a los hijos, y responsabilidades económicas de otra índole, todo lo cual consistió en una dificultad añadida a la hora de realizar labores académicas:

"Muchas veces me tocó no dormir, imagínese, trabajaba de 7:00 am a 5:00 pm, luego llegaba a la casa a atender a mis hijos y por ahi de las 10:00 pm iniciaba con mis tareas académicas".

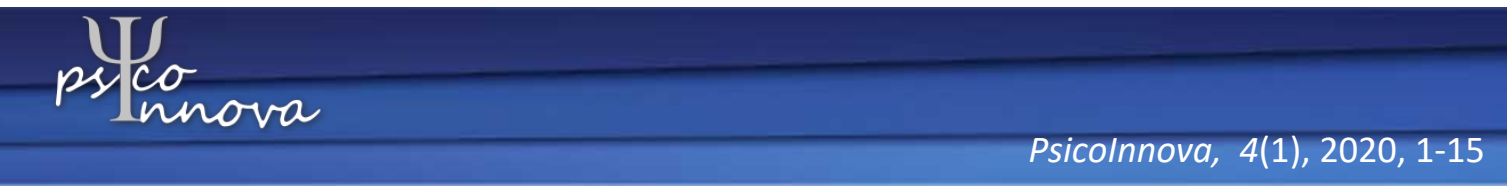


También comentan haberse sentido inseguros de sus capacidades para estudiar una carrera profesional ya que en su mayoría, ellos fueron los únicos escolarizados de sus familias y por ende sentían que tenían mucho qué probar. Resulta importante de igual forma, enfatizar en que para realizar sus estudios universitarios, todos los participantes recurrieron a préstamos.

\section{Familia.}

El tema de la familia tiene diferentes aristas, por un lado el hacinamiento familiar fue una situación compartida entre los participantes, lo cual limita el espacio personal. A pesar de lo anterior, los entrevistados expresan que el apoyo y el buen ambiente familiar facilitaron sobrellevar las condiciones de vida familiares que resultaron adversas. La figura materna fue considerada de fundamental importancia para los entrevistados, fungiendo como autoridad, apoyo y comprensión ya que era ésta la que estaba generalmente en el hogar. La idea de seguir estudiando y progresar para ayudar a la familia en el futuro, fue principalmente fomentada por la madre:

"Mi madre muchas veces me dijo, acuérdese que la familia está para apoyarse, si mañana usted está mejor, todos estaremos mejor".

La mayoría de los participantes consideraron que sus padres les fomentaron valores fundamentales como el trabajo, el esfuerzo y la honestidad, los cuales manifiestan, les han servido para crecer y superarse como personas y profesionales hoy en día.

Mientras que los participantes realizaban sus estudios universitarios la situación familiar se complicaba por el hecho de que tenían que colaborar económicamente en sus hogares o salir de la casa familiar.

En cuanto a la relación filial durante esta etapa, se mencionaron complicaciones en la relación debido a que los participantes de la investigación eran vistos por sus hermanos como presuntuosos, malagradecidos y engreídos, ya que al tener una carrera profesional o estar optando por una, se sentían "más" que ellos. Algunos de los participantes mencionan que todavía en la actualidad estos sostienen cierta desavenencia, por lo que muchos de los participantes han decidido llevar una relación de lejanía

"Mis hermanos me decían; mientras usted estaba leyendo libros y estudiando nosotros hacíamos el trabajo duro de ir a las calles y traer comida para que no se pasara hambre, y ahora que usted está bien no nos apoya como debería".

En su mayoría, los entrevistados ven como una obligación ayudar a sus familias pero les gustaría que se viera más como algo que a ellos les nace hacer, y no como que el no hacerlo consistiría en ser malagradecidos

"Mi mamá me dijo: ¿Cómo que se va de la casa? ¿Y entonces ya no me va a ayudar más después de todo lo que yo hice por usted y sus estudios? ¿Ahora que ya tiene todo y nosotros nos quedamos aqui?'”.

\section{pesco




\section{Pares y amigos.}

Los participantes coinciden en que en sus casos, hacer amigos era tarea difícil, por el hecho de que no encontraban muchas personas que tuvieran sus mismos intereses, o bien que fueran "buenas influencias"; esto debido principalmente a los altos niveles de criminalidad que existían en los barrios de origen y a las exigencias del estudio. Todos los participantes perciben a su grupo de pares de aquel entonces como gente que no se logró realizar o superar, puesto que consideran que un porcentaje muy bajo de éstos hoy en día tiene un trabajo estable y honrado o sacó una carrera profesional.

"Prácticamente todos mis amigos de mi adolescencia en este momento se encuentran en las calles, consumiendo drogas o hasta en la cárcel".

Para la época universitaria, los participantes mencionan que debido al hecho de tener que estar trabajando y estudiando al mismo tiempo, se les dificultaba tener amistades. También mencionan que crecer en un ambiente hostil los hizo un poco introvertidos y esto les dificultó el contacto. Asímismo, mencionan algunos participantes que otro posible motivo de la falta de relaciones interpersonales, podría deberse a que la familia ocupa el primer plano de prioridad por sobre las amistades; por lo que mantener a la familia estable, a aquello verdaderamente significativos para ellos, tuvo como consecuencia el alejamiento de los amigos.

\section{Trabajo.}

La mitad de los entrevistados tuvo que trabajar durante su niñez y adolescencia para poder colaborar en el hogar y continuar con los estudios. Algunos de los trabajos mencionados por los participantes fueron: carnicero, vendedor de frutas, vendedor de números o lotería y labores de reciclaje.

Para la época adulta, los participantes comentan que el trabajo fue el medio para salir adelante con los estudios y ayudar a sus familias. En la actualidad los entrevistados dicen sentirse muy satisfechos por lo que han logrado a nivel laboral, y consideran que su esfuerzo ha sido recompensado y es palpable hasta el momento, lo que les da gran satisfacción. La percepción de los entrevistados es la de personas exitosas que no sólo han logrado movilidad social ascendente, sino que además consideran que es posible seguir creciendo.

\section{Características individuales.}

Dentro de las características individuales que permitieron el ascenso social en esta muestra de adultos, se encuentra la perseverancia, la resiliencia, la constancia, el gusto por el estudio, la responsabilidad, la honradez, la autonomía, los valores morales inculcados, la puntualidad y la honestidad.

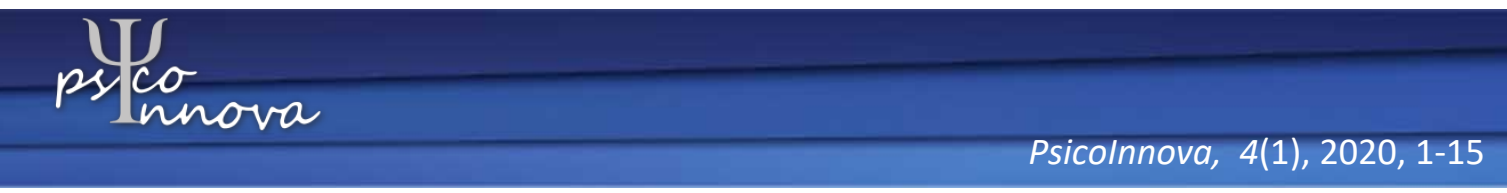


"Yo siempre fui pobre, pero la honradez me ayudó a no perder el rumbo", "Yo creo que siempre tuve la capacidad de aprender de mis errores y eso me ayudó a estar en donde estoy hoy".

Se evidencia dentro del discurso de los entrevistados, una auténtica percepción de pobreza que tenían que sobrellevar. La totalidad de los participantes mencionan que se concebían como realmente pobres, que experimentaban necesidades y que habitaban casas humildes. La mayoría afirmó que aunque no "pasaron hambre", existían muchos problemas económicos, lo cual a su vez resultó de gran motivación para lograr la movilidad social.

En su totalidad, los participantes albergaban de manera muy consciente el querer salir del barrio marginal aspirando a uno menos peligroso. Todos los entrevistados, afirman que contaron con un trabajo estable y bien remunerado en el momento del traslado con el cual pudieron hacer frente a este cambio:

"Yo me pasé de casa con mi primer trabajo. No estaba ganando tan mal, y yo no quería seguir viendo a la misma gente, estarme cuidando de [la gente peligrosa]; así que me fui a vivir al Barreal de Heredia. Imagínese; al otro lado, porque yo vivía en Hatillo".

Esto evidencia el deseo de los participantes de buscar la forma de salir del barrio originario en donde vivían buscando una vida menos peligrosa y más sana. A pesar de su problemática económica desde la niñez, los participantes indican que desde estas etapas tempranas existía el deseo de buscar un barrio más sano para vivir, lo cual desarrollaron con éxito.

\section{Discusión}

La aseveración de uno de los participantes en cuanto a que hoy en día, a excepción de un pequeño porcentaje, la gran mayoría de sus conocidos del barrio de origen no sólo carecen de un trabajo estable u honrado, sino que además muchos de ellos se encuentran ya sea en la cárcel o consumiendo drogas, preocupa y llama poderosamente la atención, en tanto el crecer bajo estas condiciones urbano-marginales consista en alguna medida en una predeterminación de vida, que no sólo condene a menores ingresos económicos, dificultades de acceso, y oportunidades de estudio; sino también en una importante medida a conductas de riesgo.

Considerando que los entrevistados referían sentimientos de peligro y miedo al andar solos en las afueras de sus barrios, pareciera que los pares de influencia negativa y las conductas de riesgo son el precio a pagar por poder disfrutar de la infraestructura que ofrece el mismo lugar. No extraña entonces que los entrevistados, personas que lograron trascender su condición, describan un buen ambiente familiar; aquellos que no logran tolerar la convivencia familiar, optarían por pasar tiempo afuera con las consecuencias que esto conlleva.

\section{pstco


El ambiente y la convivencia familiar pareciera ser en este sentido clave, no sólo por el hecho de que la totalidad de los participantes refería apoyo (o al menos, una no obstrucción), sino también porque es de parte del núcleo familiar del que refieren haber asimilado valores que describen como cruciales, concretamente de los padres. Lamentablemente no se cuenta con el dato de cuántas de estas familias consistían en hogares uniparentales.

Sobre este aspecto familiar anterior y en relación a la educación en nuestro país, los entrevistados coinciden en haber tenido una educación pública que aunque les resultase de difícil aprehensión, consideran que fue de calidad, al mismo tiempo que la brecha educativa actual entre los estudiantes del sistema público y privado crece en nuestro país; lo cual es palpable tanto en las diferencias encontradas al realizar las pruebas PISA, las pruebas de bachillerato y el examen de admisión a la Universidad de Costa Rica (Jenkins, 2015), siendo que el núcleo familiar y las características del estudiante mismo, son los principales factores a la hora de explicar estas desigualdades (Fernández y del Valle, 2013).

Este último punto se ve complementado con la investigación llevada a cabo por Montero, Rojas, Zamora y Rodino (2012) en la cual utilizando un modelo de regresión multinivel para ambos tipos de instituciones (públicas y privadas) respecto a las pruebas PISA, encuentran que un $43 \%$ de la varianza de los puntajes corresponden a factores asociados a los estudiantes, mientras que un $12 \%$ a factores asociados a la institución. Es decir, a pesar de que algunos albergan la noción de que la brecha educativa entre el sistema público y privado se explica ante la diferencia de recursos entre ambos, o en cuanto a sus naturalezas administrativas, lo cierto es que los datos estadísticos respaldan que esta desigualdad se explica en mayor medida por las condiciones socioeconómicas (Fernández y del Valle, 2013).

Adicionalmente, Montero et al (2012) elaboran en dicho informe un perfil típico de un estudiante de alto rendimiento, en el cual el único factor externo o ajeno al estudiante mismo, es el de asistir a un centro educativo ubicado en un distrito con un alto índice de desarrollo social.

De igual forma no podemos dejar de lado, que tanto en la investigación de Fernández y del Valle (2013) como en la de Montero et al (2012) para el Informe del Estado de la Educación, los factores vitales y de mayor importancia dentro del rendimiento académico, consistieron en las características del estudiante mismo, aspecto ante el cual no podemos perder la perspectiva y que resalta además la importancia de la resiliencia como enfoque ante la movilidad social y de este tipo de investigaciones de un corte más cualitativo.

En este sentido, traemos a colación la investigación de Peña-Leiva (2010) en Chile, en la cual se identificaron una serie de características personales y sociales para el logro, y que encontramos a su vez en la presente investigación, puesto que la mayoría de los participantes contaban con un auto-concepto positivo el cual les ayudó a salir adelante con su formación educativa en un entorno de deserción escolar y ausentismo así como de delincuencia juvenil. Otra característica importante encontrada fue la de tener la virtud de poder contar con una visión a futuro, ya que la generalidad de los participantes tenía como

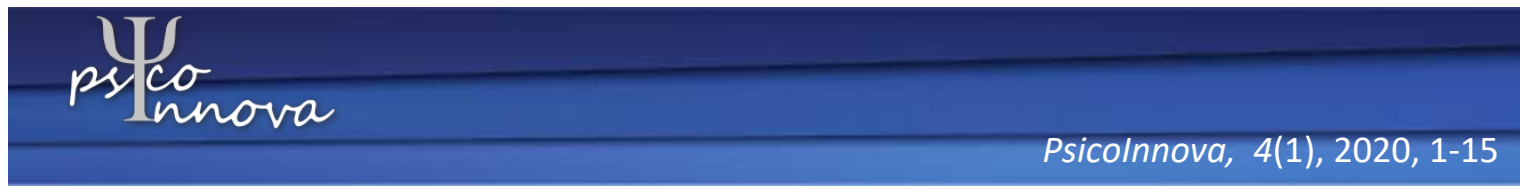


meta establecida el salir del barrio marginal en el que existían pocas oportunidades de superación así como peligrosidad, drogas y asaltos.

En lo que respecta al nivel psicosocial en Peña-Leiva (2010) también se logra respaldar los resultados encontrados, ya que la mayoría de los entrevistados experimentaron apoyo por parte de sus docentes, la exigencia sobre todo por parte de sus madres en cuanto al estudio, una visión de la educación por parte de los padres como una mejora en la calidad de vida, padres que lograban establecer límites, y también se encontró la presencia de padres que sin estudios formales hacían ver la importancia de la educación y de los valores. Adicionalmente se encontró como característica en los participantes el contar con una identidad formada, un sistema de creencias de superación basado en el estudio como medio de logro, y un fuerte convicción hacia el valor del esfuerzo y la responsabilidad.

Finalmente es importante apuntar desde la perspectiva de posibles abordajes al tema, que los participantes manifestaron la reiterada presencia de sentimientos de culpa debido a comentarios y opiniones de personas que formaban parte de sus mismas redes de apoyo y que dificultaron sus procesos de movilidad social. Pareciera ser este en consecuencia, un tema importante para trabajar a nivel comunitario en este tipo de población, sin dejar de lado creencias como la de que los hombres son los que deben llevar el sustento al hogar y las mujeres entregarse a los quehaceres del hogar, la creencia de que en la familia en cuestión "no sirven para el estudio", o situaciones de violencia, violación entre otros que afectaron a algunos de los participantes en un sentido emocional y psicológico.

\section{Conclusiones}

La presente investigación se propuso identificar y analizar distintos factores psicosociales que le permitieron a estos 8 participantes de barrios urbano-marginales lograr en la opinión de estos, superar sus estratos sociales de origen. Dentro del entorno social de los participantes, la familia como un espacio de interacción positivo en el cual no hay una desvalorización la educación resultó ser un elemento fundamental; concretamente la figura de los padres y sobre todo la figura de la madre en lo que respecta a valores, exigencias de estudio y el establecimiento de límites en el hogar.

En un sentido contrario, los pares resultaron ser por lo general una influencia negativa que no sólo no lograron trascender sus condiciones de origen, sino que además acabaron con estilos de vida desadaptados. Como consecuencia, algunos entrevistados externaron un sentimiento de carencia de intimidad con otras personas durante estos años, en el tanto las diferentes cargas añadidas a su condición no les permitía tener el tiempo para desarrollar vínculos estrechos. La mitad de los participantes tuvo que combinar trabajo con estudio aún desde etapas escolares para cooperar con las necesidades de sus familias, las cuales tendían a percibir el estudio por parte de los hijos como un medio a largo plazo de obtener un retorno económico, lo cual genera cierto malestar en algunos de los participantes aún décadas después. Estas situaciones aunadas al tener que adquirir préstamos en etapas de educación superior significa una presión superior a la normal en dichas personas que sin embargo 
lograron trascender sus estratos socioeconómicos. Dicha resiliencia se vio reflejada en los participantes por una firme voluntad de querer salir del barrio marginal de origen, en la perseverancia y constancia en aquellas acciones que los llevaran a dicha meta, en el gusto por el estudio, y en valores transversales en sus vidas de responsabilidad, puntualidad, honestidad, honradez y autonomía.

\section{Referencias}

Adams, J., White, M., Pearce, M. S., y Parker, L. (2004). Life course measures of socioeconomic position and self reported health at age 50: prospective cohort study. Journal Of Epidemiology And Community Health, 58 (12), 1028-1029. DOI: 10.1136/jech.2004.019679

Aldridge, S. (2003). The facts about social mobility. New Economy, 10 (4), 189-193. https://doi.org/10.1046/j.1468-0041.2003.00315.x

Ahluwalia, I. B., Dodds, J. M., Baligh, M. (1998). Social support and coping behaviors of low-income families experiencing food insufficiency in North Carolina. Health Education and Behavior, 25(5), 599-612. DOI: 10.1177/109019819802500507

Bazán-Ojeda, A., Quintero-Soto, M. L., y Hernández-Espitia, A. L. (2011). Evolución del concepto de pobreza y el enfoque multidimensional para su estudio. Quivera Revista $\begin{array}{llll}\text { de Estudios } & \text { Territoriales, 207-219 }\end{array}$ https://www.redalyc.org/articulo.oa?id=401/40118420013

Blane, D., Smith, G. D., y Hart, C. (1999). Some social and physical correlates of intergenerational social mobility: Evidence from the West of Scotland Collaborative Study. Sociology, 33 (1), 169-183. https://doi.org/10.1177/S0038038599000097

Bonanno, G.A., Westphal, M., Mancini, A.D. (2011). Resilence to loss and Potential Trauma. Annual Review of Clinical Psychology, 7, 511-535.

Boyle, P. J., Norman, P., y Popham, F. (2009). Social mobility: Evidence that it can widen health inequalities. Social Science \& Medicine, 68 (10), 1835-1842. https://doi.org/10.1016/j.socscimed.2009.02.051

Brady, D. (2019). Theories of the Causes of Poverty. Annual Review of Sociology, 45, 155175. https://doi.org/10.1146/annurev-soc-073018-022550

Consejo Centroamericano de Procuradores de Derechos Humanos. (2008). Políticas públicas Regionales sobre la reducción de la pobreza en Centroamérica y su incidencia en el pleno disfrute de los derechos humanos [Informe Nacional Costa Rica]. Recuperado de

https://www.iidh.ed.cr/BibliotecaWeb/Varios/Documentos.Interno/BD 125911109/ politicas_reduccion_pobreza_cr.pdf 
Desmond M, y Western B. (2018). Poverty in America: new directions and debates. Annual Reviews of Sociology, 44, 305-18

Fernández, A. y del Valle, R. (2013). Desigualdad educativa en Costa Rica: la brecha entre estudiantes de colegios públicos y privados. Análisis con los resultados de la evaluación internacional PISA. Revista Cepal, 111, 37-57. https://repositorio.cepal.org/bitstream/handle/11362/35932/RVE111FernandezDelV alle_es.pdf?sequence $=1 \&$ isAllowed $=y$

Fernández-Guzmán, E. (2019). Aspectos socioeconómicos y culturales de la movilidad social ascendente. El caso de los hijos de migrantes de Huandacareo, Michoacán, que estudiaron la universidad en Estados Unidos. Cimexus, 14(2), 173-197. https://doiorg.ezproxy.sibdi.ucr.ac.cr/10.33110/cimexus140211

González-Arratia, N., Valdez-Medina, J.L., y Zavala-Borja, Y. (2008). Resiliencia en adolescentes mexicanos. Enseñanza e Investigación en Psicología, 13(1), 41-52. Recuperado de: http://www.redalyc.org/articulo.oa?id=29213104

Guzmán, J. M., Huenchuan, S. y Montes de Oca, V. (2003). Redes de apoyo social de las personas mayores: marco conceptual. Notas de Población, 29 (77), 35-70. https://repositorio.cepal.org/bitstream/handle/11362/12750/np77035070_es.pdf?seq uence $=1 \&$ is Allowed $=\mathrm{y}$

Hernández, R., Fernández, C. y Baptista, P. (2014) Metodología de la investigación. (6ta ed). México: McGraw- Hill.

Huerta, J.E. (2012). El rol de la educación en la movilidad social de México y Chile. ¿La desigualdad por otras vías? Revista Mexicana de Investigación Educativa, 17(52), 6588. Recuperado de http://www.redalyc.org/pdf/140/14023076004.pdf

Instituto de Investigaciones en Ciencias Económicas. (2020). Estimación de los efectos del COVID-19 sobre la economía costarricense. [Universidad de Costa Rica]. http://www.iice.ucr.ac.cr/conferencia_prensa/Covid\%20IICE\%20V5-final.pdf

Instituto Nacional de Estadística y Censos. (2020). Encuesta continua de empleo. https://www.inec.cr/encuestas/encuesta-continua-de-empleo

Jenkins, M. (31 de Marzo, 2015). Crece la brecha en la educación secundaria. La Nación. https://www.nacion.com/opinion/foros/crece-la-brecha-en-la-educacionsecundaria/3ZXGQRAPXFDGHLFZTM6SCYTQDI/story/

Lomnitz, L. (1981). Cómo sobreviven los marginados. México: Siglo XXI.

López Torres, V. (2010). Educación y resiliencia: alas de la transformación social. Actualidades investigativas en educación, 2(10), 1-14. Recuperado de https://revistas.ucr.ac.cr/index.php/aie/article/view/10130/17982 
Luthar, S., Cicchetti, D. (2000). The construct of resilience: A critical evaluation and guidelines for future work. Child Development, 71(3), 543-562. https://www.ncbi.nlm.nih.gov/pmc/articles/PMC1885202/

Mason, J. (2018). Qualitative Researching. (3a Ed). London: SAGE Publications.

Montero, E., Rojas, S., Zamora, E., y Rodino, A.M. (2012). Costa Rica en las pruebas PISA 2009 de competencia lectora y alfabetización matemática. [Informe Final, Cuarto Informe del Estado de la http://repositorio.conare.ac.cr/handle/20.500.12337/861

Munist, M., Santos, H., Kotlibrenco, M., Suarez, E., Infante, F. y Grotberg, E. (1998). Manual de identificación y promoción de la resiliencia con niños y Adolescentes. [Organización Panamericana de la Salud]. Recuperado de http://resilnet.uiuc.edu/library/resilman/resilman.pdf

Neelsen, J. P. (1975). Education and Social Mobility. Comparative Education Review, 19(1), pp. 129-143

Ong Puay Tee, Ong Puay Liu, Sivapalan Selvadurai, Ong Puay Hoon, y Marsitah Mohd Radzi. (2017). Education and social mobility: perspectives of students in selected national schools in four states in Malaysia. Journal of Social Sciences and Humanities, 12(1), 78-89. http://ejournal.ukm.my/ebangi/article/view/18297

Parker, S. (1991). Diccionario McGraw Hill de Física [tomo 2]. McGrawHill.

Peña-Leiva, V. (2010). Estudio de la resiliencia como factor incidente en el desarrollo de competencias en personas de estratos socioeconómico bajo que lograron movilidad social ascendente en Chile. (Tesis inédita de Licenciatura en Psicología). Universidad de Chile. Recuperado de http://repositorio.uchile.cl/tesis/uchile/2010/cspena_v/pdfAmont/cs-pena_v.pdf

Pérez, H. (2004). Educación, capital humano y movilidad social en Costa Rica. Un primer análisis de los datos del Censo del 2000. [Capítulo de libro] http://www.kerwa.ucr.ac.cr/handle/10669/80974

Rutter, M. (1993). Resilience; some conceptual considerations. Journal of Adolescent Health, 14, 626-631.

Sagrario, G. V., Montes de Oca, V., y Arroyo M. C. (2019). Redes de apoyo en los hogares con personas adultas mayores en México. Revista Latinoamericana de Población, 14(26), 70-88. http://doi.org/10.31406/relap2020.v14.i1.n2

Sistema de Información de Tendencias Educativas en América Latina. (s.f). La transmisión intergeneracional de las desigualdades educativas. Sistema de Información de Tendencias Educativas en América Latina. Recuperado de http://www.siteal.iipeoei.org/cuadernos/13/educacion-y-movilidad-social

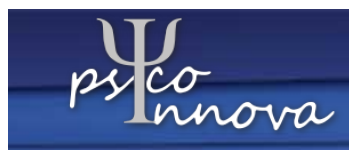


Smith, G. D., Hart, C., Watt, G., Hole, D., y Hawthorne, V. (1998). Individual social class, area-based deprivation, cardiovascular disease risk factors and mortality: The Renfrew and Paisley study. Journal of Epidemiology and Community Health, 52 (6), 399-405. https://www.ncbi.nlm.nih.gov/pmc/articles/PMC1756721/

Sokołowska, K. (2014). Determinants and perception of social mobility in Poland, 19922008. Contemporary Economics, 8(1), 89-102. https://papers.ssrn.com/sol3/papers.cfm?abstract_id=2435121

Spicker, P. (1999). Definitions of poverty: twelve clusters of meaning. http://rszarf.ips.uw.edu.pl/welfare-state/spicker.pdf

Universidad Nacional de Noreste. (2010). Clase social y Nivel Socioeconómico. [Cátedra de Atención Primaria de la Salud, epistemología e informática II. Facultad de Medicina] https://med.unne.edu.ar/sitio/multimedia/imagenes/ckfinder/files/files/aps/Clase $\% 2$ 0social $\% 20 \mathrm{y} \% 20 \mathrm{Nivel} \% 20$ Socio $\% 20-\% 20 \mathrm{Econ} \% \mathrm{C} 3 \% \mathrm{~B} 3$ mico.pdf

Villalobos-Cano, A. (2009). Desarrollo de una medida de resiliencia para establecer el perfil psicosocial de los adolescentes resilientes que permita poner a prueba el modelo explicativo de resiliencia basada en variables psicosociales (Tesis de Posgrado en Psicología Clínica). Universidad de Costa Rica, San José, Costa Rica. Recuperado de http://www.binasss.sa.cr/bibliotecas/bhp/textos/tesis11.pdf

Werner, E.E. (1984) Resilient children. Young Children,40 (1), 68-72. 